\title{
On the Stability of Thomson's Vortex $N$-gon and a Vortex Tripole/Quadrupole in Geostrophic Models of Bessel Vortices and in a Two-Layer Rotating Fluid: a Review
}

\author{
L. G. Kurakin, I. V. Ostrovskaya
}

In this paper the two-layer geostrophic model of the rotating fluid and the model of Bessel vortices are considered. Kirchhoff's model of vortices in a homogeneous fluid is the limiting case of both of these models. Part of the study is performed for an arbitrary Hamiltonian depending on the distances between point vortices.

The review of the stability problem of stationary rotation of regular Thomson's vortex $N$-gon of identical vortices is given for $N \geqslant 2$. The stability problem of the vortex tripole/quadrupole is also considered. This axisymmetric vortex structure consists of a central vortex of an arbitrary intensity and two/three identical peripheral vortices. In the model of a two-layer fluid, peripheral vortices belong to one of the layers and the central vortex can belong to either another layer or the same.

The stability of the stationary rotation is interpreted as orbital stability (the stability of a one-parameter orbit of a stationary rotation of a vortex system). The instability of the stationary rotation is instability of equilibrium of the reduced system. The quadratic part of the Hamiltonian and eigenvalues of the linearization matrix are studied.

Received June 20, 2019

Accepted November 05, 2019

L. G. Kurakin was supported by the Russian Foundation for Basic Research (Projects No. 20-55-10001) and I. V. Ostrovskaya was supported by Ministry of Education and Science of the Russian Federation, Southern Federal University (Projects No. 1.5169.2017/8.9)

Leonid G. Kurakin

kurakin@math.rsu.ru

Southern Mathematical Institute, Vladikavkaz Scientific Center of RAS

ul. Markusa 22, Vladikavkaz, 362027 Russia

Water Problems Institute of RAS

ul. Gubkina 3, Moscow, 119333 Russia

Irina V. Ostrovskaya

ivostrovskaya@sfedu.ru

Southern Federal University

ul. Milchakova 8a, Rostov-on-Don, 344090 Russia

RUSSIAN JOURNAL OF NONLINEAR DYNAMICS, 2019, 15(4), 533-542 
The parameter space is divided into three parts: $\mathbf{A}$ is the domain of stability in an exact nonlinear setting, $\mathbf{B}$ is the linear stability domain, where the stability problem requires nonlinear analysis, and $\mathbf{C}$ is the instability domain.

In the stability problem of a vortex multipole, another definition of stability is used; it is the stability of an invariant three-parametric set of all trajectories of the families of stationary orbits. It is shown that in the case of non zero total intensity, the stability of the invariant set implies orbital stability.

Keywords: $N$-vortex problem, Thomson's vortex $N$-gon, point vortices, two-layer fluid, stability, Hamiltonian equation

\section{The stability of the Thomson vortex $N$-gon for a system of particles with potential of interaction depending on the distances between them}

We consider the motion of the system of $N$ equal point vortices with intensity $\Gamma \neq 0$ described by the Hamiltonian depending on the distances between vortices

$$
\mathcal{H}=-\frac{\Gamma}{4 \pi} \sum_{1 \leqslant j<k \leqslant N} V\left(\left|z_{j}-z_{k}\right|\right)
$$

where $z_{k}=q_{k}+i p_{k},\left(q_{k}, p_{k}\right)$ are Cartesian coordinates of the $k$ th vortex and $V(\xi)$ is a smooth function defined on $\mathbb{R}_{+}$.

The form of function $V(\xi)$ defines the vortex dynamics model under study. The formula

$$
V(\xi)=\ln \xi
$$

defines Kirchhoff's model describing the motion of point vortices in a homogeneous fluid. In the case

$$
V(\xi)=-K_{0}(\gamma \xi)
$$

we have a model of the geostrophic Bessel vortices [11, 14, 19, 20], and if

$$
V(\xi)=\ln \xi-\frac{h_{1}}{h_{2}} K_{0}(\gamma \xi)
$$

the geostrophic vortices are located in the two-layer rotating fluid [4, 18]. Here $K_{0}(\xi)$ is a modified Bessel function of zero order, $1 / \gamma>0$ is the internal Rossby deformation radius, and $h_{1}, h_{2}$ are the thickness of the top and bottom layer, respectively, $h_{1}+h_{2}=1$. The case $\gamma \neq 1$ can be reduced to the case $\gamma=1$ by scaling. Note that function (1.2) is the limit for functions (1.3) and (1.4) as $\gamma \rightarrow 0$ and $h_{1} \rightarrow 0$, respectively.

We consider the stability problem of the stationary rotation of a system of $N$ identical point vortices lying uniformly on a circle of radius $R$. In the two-layer model the vortices are located in a single layer of a two-layer fluid (for example, in the bottom layer).

The system with Hamiltonian (1.1) has the exact solution

$$
z_{k}=e^{i \omega_{N} t} u_{k}, \quad u_{k}=R e^{2 \pi i(k-1) / N}, \quad R>0, \quad k=1, \ldots, N,
$$


corresponding to the stationary rotation of the configuration of $N$ point vortices lying on a circle of radius $R$ at the vertices of the regular $N$-gon. The angular velocity $\omega_{N}$ is given by the expression

$$
\omega_{N}=\frac{\Gamma}{4 \pi R} \sum_{m=1}^{N-1} V^{\prime}\left(2 R \sin \frac{m \pi}{N}\right) \sin \frac{m \pi}{N} .
$$

The stability of the stationary motion is interpreted as orbital stability.

The stability problem of the stationary rotation of the system of $N$ identical point vortices located uniformly on a circle (Thomson's vortex $N$-gon) in a homogeneous fluid (1.2) was posed by Kelvin (W. Thomson) [15]. J. J. Thomson [16] and T. H. Havelock [5] investigated this problem completely in the linear setting. A long history of research on this problem in the exact nonlinear setting for Kirchhoff's model is described in [1,8], where a review of experimental and theoretical works is given.

The change of variables

$$
z_{k}(t)=e^{i \omega_{N} t} v_{k}(t), \quad v_{k}=\sqrt{R^{2}+2 r_{k}} e^{i\left(\frac{2 \pi}{N}(k-1)+\theta_{k}\right)}, \quad k=1,2, \ldots, N
$$

leads the Hamiltonian (1.1) to perturbation equations with the Hamiltonian

$$
\begin{aligned}
E(\boldsymbol{\rho}) & =\mathcal{H}(\boldsymbol{\rho})+\frac{\omega_{N}}{2} M(\boldsymbol{\rho}), \quad M=\sum_{k=1}^{N}\left(R^{2}+2 r_{k}\right), \\
\boldsymbol{\rho} & =\left(r_{1}, \ldots, r_{N}, \theta_{1}, \ldots, \theta_{N}\right) .
\end{aligned}
$$

The equilibrium family $\mathcal{C}=\left\{r_{1}=\ldots=r_{N}=0, \theta_{1}=\ldots=\theta_{N}\right\}$ corresponds to stationary rotation (1.5). The stability of this family is the orbital stability of stationary rotation. The instability of the stationary rotation of an $\mathrm{N}$-gon (1.5) is the instability of an invariant twodimensional set $\mathcal{C}_{2}$ of all orbits of a continuous family of stationary rotations (1.5) depending on $R$ :

$$
\mathcal{C}_{2}=\left\{\boldsymbol{\rho} \in \mathbb{R}^{2 N}: r_{1}=\ldots=r_{N}, \theta_{1}=\ldots=\theta_{N}\right\}
$$

or Lyapunov instability of zero equilibrium of the reduced system.

The quadratic part of the Taylor series expansion of the Hamiltonian $E$ in powers of $\rho$ in the neighborhood of the zero solution has the form

$$
E_{2}(\boldsymbol{\rho})=(\mathbf{S} \boldsymbol{\rho}, \boldsymbol{\rho}), \quad \mathbf{S}=\left(\begin{array}{cc}
\mathbf{F}_{1} & \mathbf{G}_{0} \\
-\mathbf{G}_{0} & \mathbf{F}_{2}
\end{array}\right)
$$

The linearization matrix is

$$
\mathbf{L}=2\left(\begin{array}{cc}
-\mathbf{G}_{0} & \mathbf{F}_{2} \\
-\mathbf{F}_{1} & -\mathbf{G}_{0}
\end{array}\right) .
$$

The matrices $\mathbf{F}_{1}, \mathbf{F}_{2}$ and $\mathbf{G}_{0}$ are circulants

$$
\mathbf{F}_{j}=\sum_{m=0}^{N-1} f_{j m} \mathbb{C}^{m}, \quad \mathbf{G}_{0}=\sum_{m=1}^{N-1} g_{0 m} \mathbb{C}^{m} .
$$

The analytical formulas of coefficients $f_{j m}$ and $g_{0 m}$ for an arbitrary Hamiltonian are given by formulas (3.15), (3.16) of [12]. The cyclic matrix $\mathbb{C}=\left\{c_{j k}\right\}_{j, k=1}^{N}$ has nontrivial coefficients, namely, only those above its principal diagonal $c_{1,2}=\ldots=c_{N-1, N}=1$ and in the left lower corner $c_{N, 1}=1$. 
Analysis of eigenvalues of matrices $\mathbf{S}$ and $\mathbf{L}$ gives the following statements [12].

A The Thomson $N$-gon (1.5) is orbitally stable in an exact nonlinear setting if all eigenvalues of matrix $\mathbf{S}$ have identical sign, except a simple zero.

C The Thomson $N$-gon (1.5) is instable if the linearization matrix $\mathbf{L}$ has at least one eigenvalue in the right half-plane.

B The stability problem requires nonlinear analysis if conditions $\mathbf{A}$ and $\mathbf{C}$ are not valid.

In the case of the Kirchhoff model the rotation of the vortex $N$-gon is stable only if $N \leqslant 7$, whereas for $N \geqslant 8$ the motion is unstable $(\mathbf{C})[5,8]$. In the case $N \leqslant 6$ a linear analysis is enough to conclude nonlinear stability (A). For $N=7$, it is necessary to involve nonlinear terms for analysis of the stability [8].

\subsection{Thomson's $N$-gon in the geostrophic model of the Bessel vortices}

The motion of the system of $N$ point geostrophic (Bessel) vortices is described by the Hamiltonian (1.1), (1.3). The parameter space is $(N, R)$. Stewart $[19,20]$ performed a linear analysis of the stability of the regular vortex $N$-gon and came to the following conclusions: 1) Stability always occurs for $N \leqslant 6$ (there are no exponentially growing modes); 2 ) The vortex heptagon $(N=7)$ is stable if $R>71$, and unstable for $R<71 ; 3)$ Instability always occurs for $N \geqslant 8$.

Morikawa and Swenson [14] specified that the vortex hexagon $(N=6)$ is linearly stable at $R<R_{06}$ and exponentially unstable if $R>R_{06}, R_{06} \approx 1.288$.
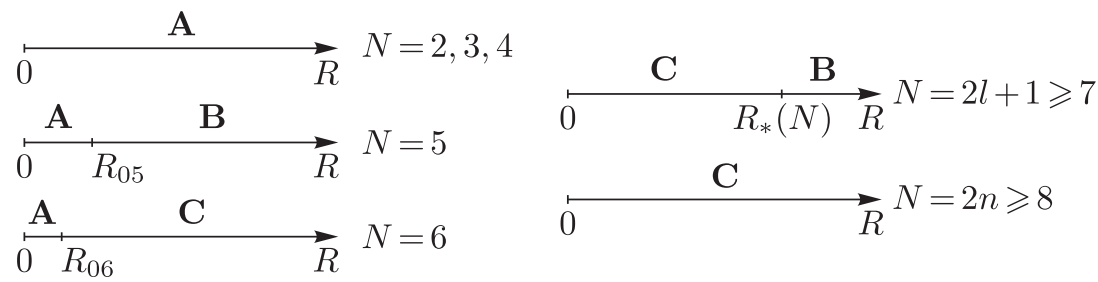

Fig. 1. Stability diagrams of the vortex $N$-gon in the model of Bessel vortices: $\mathbf{A}$ - the stability in the case of exact nonlinear problem setting, $\mathbf{B}$ - the problem requires nonlinear analysis, and $\mathbf{C}$ instability.

In [11] it is proved that the vortex $N$-gon $(N=2,3,4)$ is orbitally stable $(\mathbf{A})$ in the exact nonlinear setting for $R>0$ (see Fig. 1). The vortex pentagon $(N=5)$ is orbitally stable $(\mathbf{A})$ if $R<R_{05}, R_{05} \approx 3.76$ and the stability problem requires nonlinear analysis $(\mathbf{B})$ if $R \geqslant R_{05}$. The vortex hexagon $(N=6)$ is orbitally stable $(\mathbf{A})$ if $R<R_{06}$. In the case of odd $N=2 \ell+1 \geqslant 7$ the vortex $N$-gon is unstable $(\mathbf{C})$ if $R<R_{*}(N)$ and the stability problem requires nonlinear analysis (B) if $R \geqslant R_{*}(N)$. In the case of even $N=2 m \geqslant 8$ the vortex $N$-gon is unstable (C) for any $R>0$. The asymptotic formulas for the value $R_{*}(N)$ are

$$
R_{*}(N) \approx R_{a}(N)=\frac{1}{2 \sin ^{3} \frac{\pi}{N}}\left[\frac{2 \cos \frac{2 \pi}{N}}{1+\cos \frac{\pi(N-1)}{N}}-1\right]+\frac{1}{4 \sin \frac{\pi}{N}} .
$$




\subsection{Thomson's $N$-gon in a two-layer fluid}

The motion of $N$-point vortices in a two-layer fluid is described by the Hamiltonian (1.1), (1.4). The parameter space is $(N, R, \alpha)$. The parameter $\alpha=h_{2}-h_{1}$ is the difference between the thicknesses of the lower and upper layers, $-1<\alpha<1$.

The case $\mathbf{A}$ takes place for $N=2,3,4$ for all $R>0$ and $-1<\alpha<1$ [10].

In the case of $N=5$ we have two domains: $\mathbf{A}$ and $\mathbf{B}$ (see Fig. 2a). At $N=6$ cases $\mathbf{A}$ and $\mathbf{C}$ take place (see Fig. 2b). The case $\mathbf{B}$ is the neutral curve $\alpha_{6}$ defined for $R_{06} \leqslant R<\infty$. In the case of $N=7$ there are all three domains $\mathbf{A}, \mathbf{B}$ and $\mathbf{C}$ (see Fig. 3). The instability $\mathbf{C}$ takes place always for even $N=2 m \geqslant 8$. In the case of odd $N=2 \ell+1 \geqslant 9$ there are two domains $\mathbf{B}$ and $\mathbf{C}$ (see Fig. 4). The analytical formulas of neutral curves $\alpha_{N}$ have been obtained in [10].

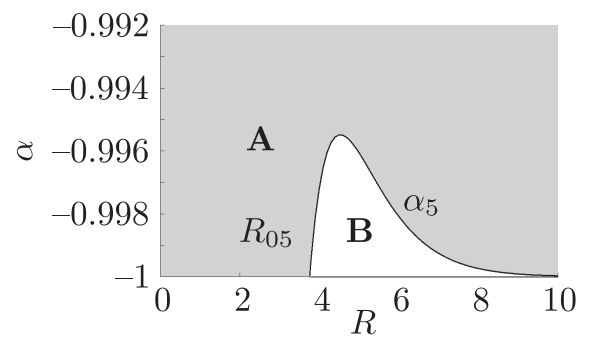

(a)

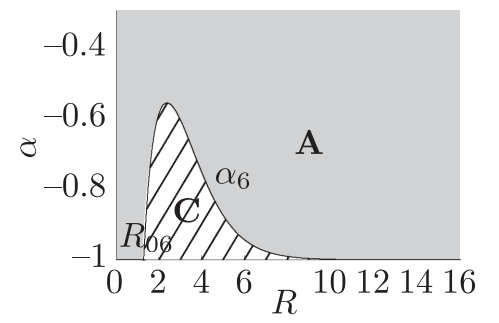

(b)

Fig. 2. Stability diagrams of a vortex $N$-gon: a) vortex pentagon; b) vortex hexagon.

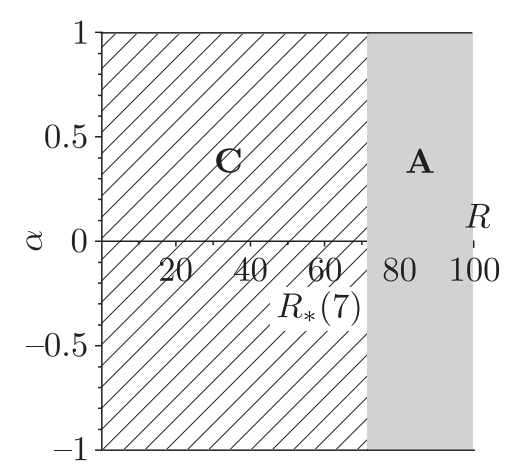

(a)

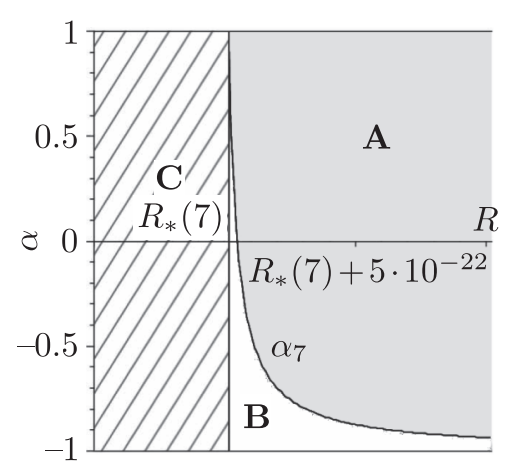

(b)

Fig. 3. Stability diagram of a vortex heptagon: a) $0<R \leqslant 100$, b) $\left|R-R_{*}(7)\right|<10^{-21}$, $R_{*}(7) \approx 71.52720252$.

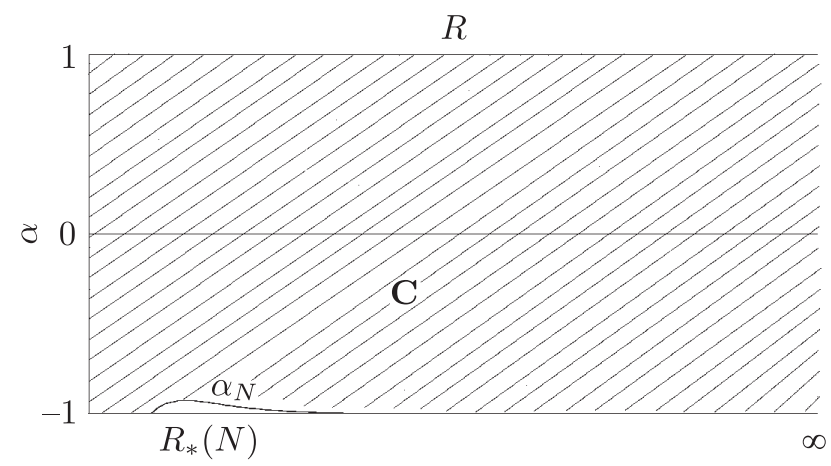

Fig. 4. The schematic diagram of stability for an $N$-gon for odd $N=2 \ell+1 \geqslant 9 . R_{*}(N)$ is given by (1.11). 


\section{The stability problem for a vortex tripole and a vortex quadrupole}

\subsection{The model of a homogenous fluid (Kirchhoff's equation)}

The motion of the system of one vortex with arbitrary intensity $\Gamma \neq 0$ and $N$ vortices of unit intensity is described by the Hamiltonian

$$
\begin{aligned}
\mathcal{H}= & -\frac{1}{4 \pi}\left[\Gamma \sum_{k=1}^{N} \ln \sqrt{\left(q_{0}-q_{k}\right)^{2}+\left(\frac{p_{0}}{\Gamma}-p_{k}\right)^{2}}+\right. \\
& \left.+\sum_{1 \leqslant j<k \leqslant N} \ln \sqrt{\left(q_{j}-q_{k}\right)^{2}+\left(p_{j}-p_{k}\right)^{2}}\right] .
\end{aligned}
$$

The system has the first integrals: the Hamiltonian (2.1)

$$
\begin{aligned}
I_{1} & =\Gamma q_{0}+\sum_{k=1}^{N} q_{k}, \quad I_{2}=\sum_{k=0}^{N} p_{k}, \\
M & =\Gamma q_{0}^{2}+\frac{p_{0}^{2}}{\Gamma}+\sum_{k=1}^{N}\left(q_{k}^{2}+p_{k}^{2}\right), \quad \mathcal{L}=(\Gamma+N) M-I_{1}^{2}-I_{2}^{2} .
\end{aligned}
$$

In complex variables $z_{k}=q_{k}+i p_{k}$, the problem has the exact solution

$$
\begin{aligned}
z_{k}(t) & =e^{i \omega_{N} t} u_{k}, \quad u_{0}=0, \quad u_{k}=R e^{i \frac{2 \pi}{N}(k-1)}, \quad k=1,2, \ldots, N, \\
\omega_{N} & =\frac{N-1+2 \Gamma}{4 \pi R^{2}} .
\end{aligned}
$$

corresponding to stationary rotation of configuration of a central vortex of intensity $\Gamma$ and $N$ vortices of equal intensity lying uniformly on a circle of radius $R$.

The orbital stability is the stability of a one-parameter orbit of a stationary rotation of a vortex multipole (2.2).

The vortex tripole $(N=2)$ is linearly stable if $\Gamma<-\frac{5}{4}$, and unstable $(\mathbf{C})$ if $\Gamma>-\frac{5}{4}[13]$. The vortex quadrupole $(N=3)$ is linearly stable if $\Gamma<1$ and unstable $(\mathbf{C})$ if $\Gamma>1$ [3]. The vortex quadrupole $(N=3)$ is orbitally stable if $0<\Gamma<1$, the problem requires nonlinear analysis if $\Gamma<0[9,10]$. To our surprise, we have found no earlier references to nonlinear stability analysis. The proof and the nonlinear stability condition given in [2] are incorrect.

Let $\mathfrak{M}_{N}$ be the invariant three-parametric set of all trajectories of the families of stationary orbits

$$
z_{k}(t)=u_{k} e^{i \omega_{N} t}+\eta, \quad k=0, \ldots, N, \quad \eta \in \mathbb{C} .
$$

Z. Kizner [6,7] used the following definition of stability: the vortex multipole is steady if, at a reasonably small initial perturbation of distances between its vortices, these distances remain weakly perturbed for all time. Such stability is stability of the invariant set $\mathfrak{M}_{N}$.

The invariant set $\mathfrak{M}_{N}$ is nonlinearly stable if the first integral of the system exists which has a local extremum on the set $\mathfrak{M}_{N}$.

To study the stability of the invariant set $\mathfrak{M}_{N}$, we introduce new variables $X_{1}, X_{2}, \Theta_{2}$ for $N=2$ or $X_{1}, X_{2}, X_{3}, \Theta_{2}, \Theta_{3}$ for $N=3$ (see Fig. 5). 

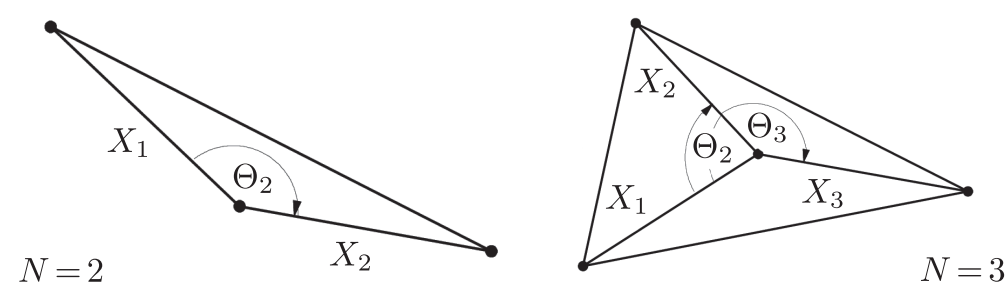

Fig. 5

If $N=2$, the system integrals $\mathcal{H}$ and $\mathcal{L}$ in the new variables are rewritten as

$$
\begin{aligned}
\mathcal{H}\left(X_{1}, X_{2}, \Theta_{2}\right) & =\Gamma\left(\ln X_{1}+\ln X_{2}\right)+\ln \sqrt{X_{1}^{2}+X_{1}^{2}-2 X_{1} X_{2} \cos \Theta_{2}}, \\
\mathcal{L}\left(X_{1}, X_{2}, \Theta_{2}\right) & =\Gamma\left(X_{1}^{2}+X_{2}^{2}\right)+X_{1}^{2}+X_{2}^{2}-2 X_{1} X_{2} \cos \Theta_{2} .
\end{aligned}
$$

The point $(R, R, \pi)$ in the new variables corresponds to the invariant set $\mathfrak{M}_{2}$. The invariant set $\mathfrak{M}_{2}$ is stable if the integral

$$
\mathcal{I}\left(X_{1}, X_{2}, \Theta_{2}\right)=\left(\mathcal{H}\left(X_{1}, X_{2}, \Theta_{2}\right)-\mathcal{H}_{*}\right)^{2}+\left(\mathcal{L}\left(X_{1}, X_{2}, \Theta_{2}\right)-\mathcal{L}_{*}\right)^{2}
$$

has a strict local minimum at the point $(R, R, \pi), \mathcal{H}_{*}=\mathcal{H}(R, R, \pi), \mathcal{L}_{*}=\mathcal{L}(R, R, \pi)$.

The invariant set $\mathfrak{M}_{2}$ is stable if $\Gamma<-\frac{5}{4}$ (see $[6,7]$ ), and the invariant set $\mathfrak{M}_{3}$ is stable if $\Gamma<-3$ and $0<\Gamma<1[10]$.

\subsection{The model of a two-layer rotating fluid}

A set of $N+1$ point vortices in a two-layer fluid is considered for $N=2,3$ : one vortex of arbitrary intensity $\varkappa_{0} \neq 0$ with coordinates $\left(q_{0}, p_{0}\right)$ and $N$ vortices with coordinates $\left(q_{k}, p_{k}\right), k=1, \ldots, N$ with equal intensity $\varkappa$ lying in one layer, for definiteness, in the lower layer $\left(\varkappa h_{2}=1\right)$. Two cases are distinguished: $(\mathbf{I})$ the vortex of intensity $\varkappa_{0}$ is located in the upper layer with thickness $h_{1}, \Gamma=\varkappa_{0} h_{1}$; (II) all $N+1$ vortices are located in the lower layer with thickness $h_{2}, \Gamma=\varkappa_{0} h_{2}$. The motion of the vortex system is described by the Hamiltonian $\mathcal{H}[4,18]$ :

$$
\begin{aligned}
& \mathcal{H}=-\frac{1}{4 \pi}\left[\Gamma \sum_{k=1}^{N} V\left(\sqrt{\left(q_{0}-q_{k}\right)^{2}+\left(\frac{p_{0}}{\Gamma}-p_{k}\right)^{2}}\right)+\right. \\
& \left.+\sum_{1 \leqslant j<k \leqslant N} W\left(\sqrt{\left(q_{j}-q_{k}\right)^{2}+\left(p_{j}-p_{k}\right)^{2}}\right)\right], \\
& W(\xi)=\ln \xi-\frac{1-\alpha}{1+\alpha} \mathrm{K}_{0}(\xi), \quad V(\xi)=\left\{\begin{array}{lll}
\ln \xi+\mathrm{K}_{0}(\xi) & \text { in case } & (\mathbf{I}), \\
W(\xi) & \text { in case } & (\mathbf{I I}) .
\end{array}\right.
\end{aligned}
$$

A complete analysis of the stability problem of the stationary rotation (2.2) of a vortex tripole and a vortex quadrupole with constant angular velocity

$$
\omega_{2}=\frac{1}{4 \pi R}\left(\Gamma V^{\prime}(R)+W^{\prime}(2 R)\right), \quad \omega_{3}=\frac{1}{4 \pi R}\left(\Gamma V^{\prime}(R)+\sqrt{3} W^{\prime}(\sqrt{3} R)\right)
$$

is presented in $[6,7,9,10,17]$. Its results are presented in Figs. 6-9. The formulas of curves $\Gamma_{j}$, $j=1, \ldots, 6$ are obtained in $[9,10]$. 


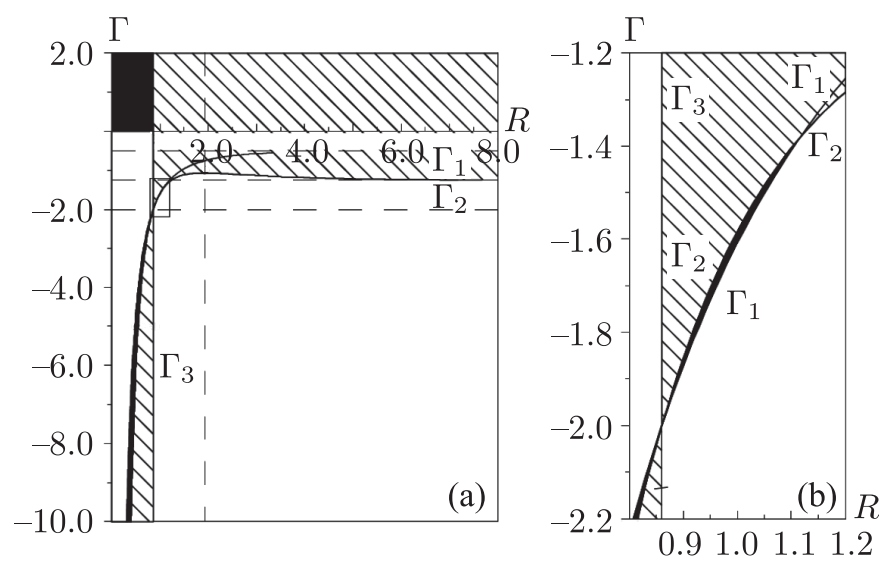

Fig. 6. Stability diagram of a vortex tripole. Case I. $\alpha=0$. $\mathbf{A}-$ dark domain, $\mathbf{B}-$ light domain, $\mathbf{C}-$ dashed domain.

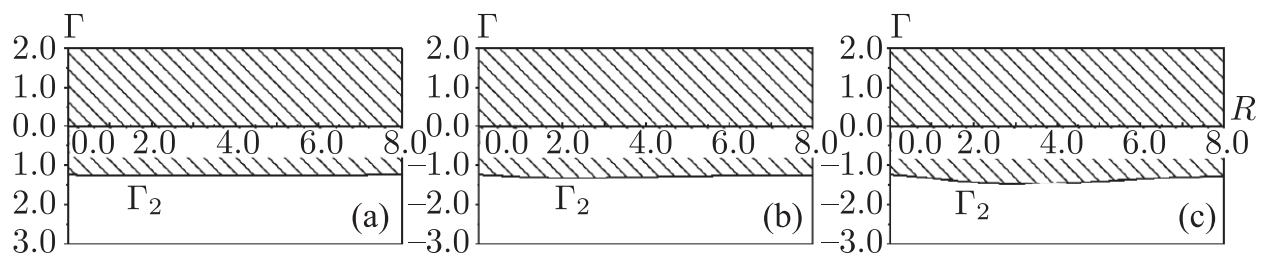

Fig. 7. Stability diagram of a vortex tripole. Case II: a) $\alpha=0.8$; b) $\alpha=0$; c) $\alpha=-0.8$.
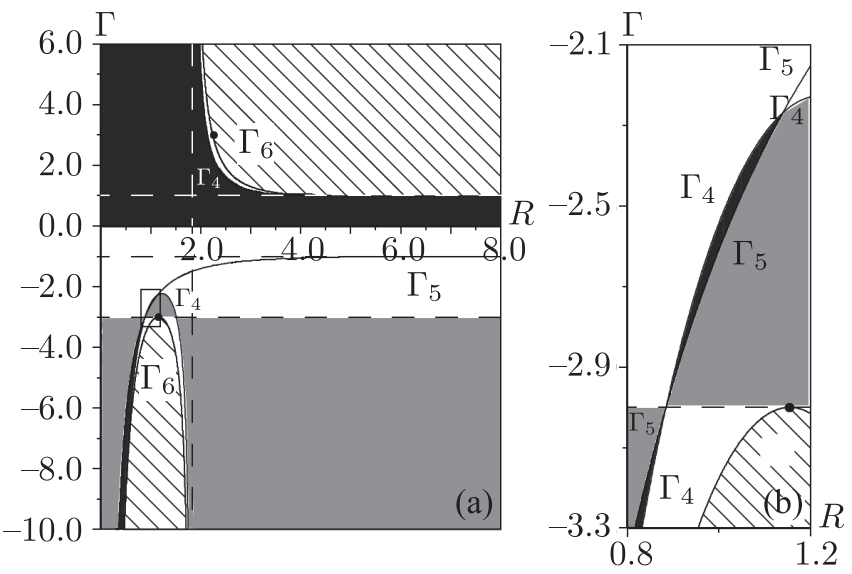

Fig. 8. Stability diagram of a vortex quadrupole. Case I: $\mathrm{a}-\mathrm{b}) \alpha=0.8$. The stability of the invariant set $\mathfrak{M}_{3}$ - grey domain.

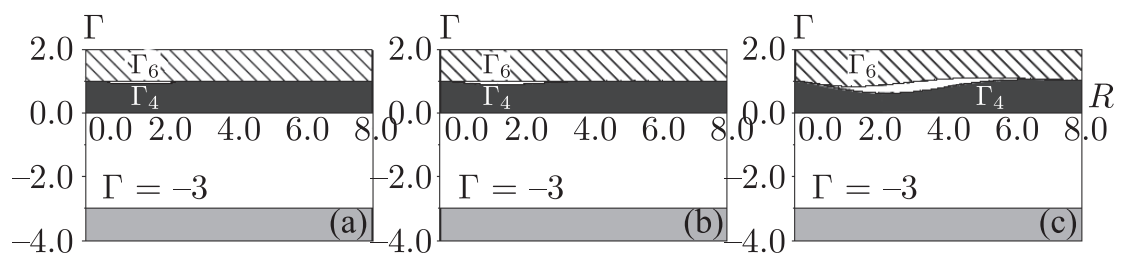

Fig. 9. Stability diagram of a vortex quadrupole. Case II: a) $\alpha=0.8$; b) $\alpha=0$; c) $\alpha=-0.8$. 
The invariant set $\mathfrak{M}_{2}$ is stable everywhere in the orbital stability domain $\mathbf{A}$ and in domain $\mathbf{B}$, where nonlinear analysis is required [6, 7].

The stability of the invariant set $\mathfrak{M}_{3}$ in the case of a two-layer fluid is studied in [10]. In the stability diagrams presented in Figs. 8, 9, the domain of stability of the invariant set $\mathfrak{M}_{3}$ consists of a dark domain of orbital stability $\mathbf{A}$ and a grey domain, where the invariant set $\mathfrak{M}_{3}$ is stable and condition $\mathbf{B}$ is valid (see Section 1).

\subsection{On equivalence of stability of invariant set and orbital stability in case of non zero total intensity}

Note that the integral $\mathcal{K}=I_{1}^{2}+I_{2}^{2}$ on set $\mathfrak{M}_{N}$ has the value

$$
\mathcal{K}=(\Gamma+N)^{2}|\eta|^{2} .
$$

The integral

$$
\mathcal{J}=\left(\mathcal{H}-\mathcal{H}_{*}\right)^{2}+\left(\mathcal{L}-\mathcal{L}_{*}\right)^{2}+\mathcal{K},
$$

where $\mathcal{H}_{*}=\left.\mathcal{H}\right|_{\mathfrak{M}_{N}}, \mathcal{L}_{*}=\left.\mathcal{L}\right|_{\mathfrak{M}_{N}}$, has a strict local minimum at $\eta=0$ on the orbit $(2.2),(2.3)$ if $\Gamma+N \neq 0$.

REMARK. In all the above cases the stability of the invariant set $\mathfrak{M}_{N}$ implies orbital stability of the stationary rotation of the vortex multipole.

\section{References}

[1] Borisov, A.V. and Mamaev, I.S., Mathematical Methods in the Dynamics of Vortex Structures, Izhevsk: R\&C Dynamics, Institute of Computer Science, 2005 (Russian).

[2] Cabral, H.E. and Schmidt, D. S., Stability of Relative Equilibria in the Problem of $N+1$ Vortices, SIAM J. Math. Anal., 1999/2000, vol. 31, no. 2, pp. 231-250.

[3] Campbell, L. J., Transverse Normal Modes of Finite Vortex Arrays, Phys. Rev. A, 1981, vol.24, no. 1 , pp. 514-534.

[4] Gryanik, V.M., Dynamics of Singular Geostrophical Vortices in a 2-Level Model of the Atmosphere (Ocean), Izv. Atmos. Ocean Phys., 1983, vol.19, no. 3, pp.171-179; see also: Izv. Akad. Nauk SSSR. Fiz. Atmos. Okeana, 1983, vol. 19, no. 3, pp. 227-240.

[5] Havelock, T.H., The Stability of Motion of Rectilinear Vortices in Ring Formation, Philos. Mag., 1931, vol. 11, no. 70, pp. 617-633.

[6] Kizner, Z., Stability of Point-Vortex Multipoles Revisited, Phys. Fluids, 2001, vol. 23, no. 6, 064104, $11 \mathrm{pp}$.

[7] Kizner, Z., On the Stability of Two-Layer Geostrophic Point-Vortex Multipoles, Phys. Fluids, 2014, vol. 26, no. 4, 046602, 18 pp.

[8] Kurakin, L. G. and Yudovich, V. I., The Stability of Stationary Rotation of a Regular Vortex Polygon, Chaos, 2002, vol. 12, no. 3, pp. 574-595.

[9] Kurakin, L. G., Ostrovskaya, I. V., and Sokolovskiy, M. A., Stability of Discrete Vortex Multipoles in Homogeneous and Two-Layer Rotating Fluid, Dokl. Phys., 2015, vol.60, no. 5, pp. 217-223; see also: Dokl. Akad. Nauk, 2015, vol.462, no.2, pp.161-167.

[10] Kurakin, L.G., Ostrovskaya, I. V., and Sokolovskiy, M. A., On the Stability of Discrete Tripole, Quadrupole, Thomson' Vortex Triangle and Square in a Two-Layer/Homogeneous Rotating Fluid, Regul. Chaotic Dyn., 2016, vol. 21, no. 3, pp. 291-334.

[11] Kurakin, L. G. and Ostrovskaya, I.V., On Stability of the Thomson's Vortex $N$-Gon in the Geostrophic Model of the Point Bessel Vortices, Regul. Chaotic Dyn., 2017, vol. 22, no.7, pp. 865-879. 
[12] Kurakin, L. G., Lysenko, I. A., Ostrovskaya, I. V., and Sokolovskiy, M. A., On Stability of the Thomson's Vortex $N$-Gon in the Geostrophic Model of the Point Vortices in Two-Layer Fluid, J. Nonlinear Sci., 2019, vol. 29, no. 4, pp. 1659-1700.

[13] Mertz, G., Stability of Body-Centered Polygonal Configurations of Ideal Vortices, Phys. Fluids, 1978, vol. 21, no. 7, pp. 1092-1095.

[14] Morikawa, G. K. and Swenson, E. V., Interacting Motion of Rectilinear Geostrophic Vortices, Phys. Fluids, 1971, vol. 14, no. 6, pp. 1058-1073.

[15] Thomson, W., Floating Magnets (Illustrating Vortex Systems), Nature, 1878, vol. 18, pp. 13-14. See also: Kelvin, W. T., Mathematical and Physical Papers: Vol. 4, Cambridge: Cambridge Univ. Press, 1910, pp. 162-164.

[16] Thomson, J. J., Treatise on the Motion of Vortex Rings, London: Macmillan, 1883, pp. 94-108.

[17] Sokolovskiy, M. A. and Verron, J., Some Properties of Motion of $A+1$ Vortices in a Two-Layer Rotating Fluid, Nelin. Dinam., 2006, vol.2, no. 1, pp. 27-54 (Russian).

[18] Sokolovskiy, M. A. and Verron, J., Dynamics of Vortex Structures in a Stratified Rotating Fluid, Atmos. Oceanogr. Sci. Libr., vol. 47, Cham: Springer, 2014.

[19] Stewart, H. J., Periodic Properties of the Semi-Permanent Atmospheric Pressure Systems, Quart. Appl. Math., 1943, vol.1, pp. 262-267.

[20] Stewart, H. J., Hydrodynamic Problems Arising from the Investigation of the Transverse Circulation in the Atmosphere, Bull. Amer. Math. Soc., 1945, vol.51, pp. 781-799. 